\title{
Improved AODV based on Load and Delay for Route Discovery in MANET
}

\author{
Shital Umredkar ${ }^{1}$, Sitendra Tamrakar ${ }^{2,}$ Umesh Kumar Lilhore ${ }^{3}$ \\ I (Department of Computer Science and Engineering, NRI Institute of Information Science and Technology, \\ Bhopal, Madhya Pradesh, India /RGPV, India) \\ ${ }^{2}$ (Department of Computer Science and Engineering, NRI Institute of Information Science and Technology, \\ Bhopal, Madhya Pradesh, India /RGPV, India) \\ ${ }^{3}$ (Department of Computer Science and Engineering, NRI Institute of Information Science and Technology, \\ Bhopal, Madhya Pradesh, India /RGPV, India)
}

\begin{abstract}
A mobile Ad-hoc network (MANET) is a self configuring network of mobile devices connected by wireless links. A dynamic traffic allocation algorithm based on packet delay and hops in Mobile Ad hoc networks is proposed. The algorithm is based on the minimization product of delay and the number of hops in each path and adjusts the traffic adaptively so as to make load-balanced which optimizes network resource utilization. Simulation demonstrated that the algorithm could dynamically balance the traffic allocation between paths. The aim of the minimum utilization of resource in mobile Ad hoc networks can be achieved.
\end{abstract}

Keywords : MANET, Node Load, Route Weight, Routing Delay, TAODV.

\section{INTRODUCTION}

Mobile Ad-Hoc Network is a complex distributed system that consists of various wireless mobile nodes which can dynamically and freely organize into temporary or "ad-hoc" network topologies, allowing devices to interconnect in areas without having any predefined communication infrastructure. In Mobile Ad-Hoc Networks, each mobile hosts acts as the router as well as host at the same time.

Figure 1 illustrates the overview of Mobile Ad-Hoc Network. In general, a wireless mobile node can act as any computing equipment that employs the air as the transmission medium. As shown, the wireless mobile node may be physically attached to a vehicle, a person, or an airplane, to enable wireless communication among them.

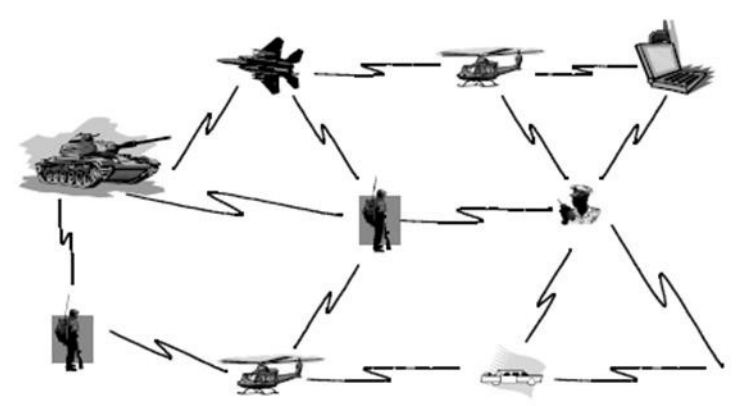

Figure.1. Overview of Mobile Ad-hoc Network

In a Mobile Ad-Hoc Network, all the mobile nodes communicate with each other to forward the packets from source to destination in the network and hence, each node is effectively acted as a router. Thus routing is one of the most important issues in MANET. In this section, some of the other issues in ad hoc networks are described. (a) Distributed network: Mobile Ad-Hoc Network is a complex distributed system that consists of various wireless mobile nodes interconnected in areas without having any predefined communication infrastructure. In Distributed system there is no centralized administration or support to the network.

(b) Dynamic topology: The nodes are mobile and free to move anywhere, hence the network is self-organizing. Due to this nature of network, the topology of the network is not constant with the time.

(c) Power awareness: As the nodes in Mobile Ad-Hoc Network typically run on batteries, they have stringent power requirements.

(d) Security: Due to Heterogeneity of nodes in MANETs, it increases the Vulnerability of MANET nodes. In a network connective links of MANET nodes are open to inspection or targeted data capture leads to decreasing Survivability. 


\subsection{Advantages of Mobile Ad hoc Networks}

(a) Low cost of deployment: Mobile Ad-Hoc Networks can be deployed on the fly, thus it not requires any expensive infrastructure such as copper wires, data cables, etc or not any central administration to support the network.

(b) Fast deployment: ad hoc networks are very convenient and easy to deploy, when it compared to WLANs because it requires less manual intervention.

(c) Dynamic Configuration: Configuration of Mobile Ad-Hoc Network can changes dynamically with time. Mobile Ad-Hoc Networks has various potential applications in various fields. In Civilian environments it includes taxi cab network, meeting rooms, sports stadium and boats, small aircrafts. In emergency operation it includes search and rescue, policing and fire fighting. The other applications of MANET includes sensor Networks, Collaborative computing, Communications in disaster recovery and areas battlefields.

\section{Previous Work:}

In [1], the schemes of finding multi-routing paths from source to destination by detecting process. By this detective process some uncross routing paths between source and destination nodes are also available this can make up the advantage over the traditional route protocol which only finds a single routing path in the detecting process. The total effective network bandwidth will be enlarged by having multiple paths. We can achieve the aim of optimizing the network performance by allocating the total traffic to each path [2]. Many routing protocols are used to manage the traffic in Ad Hoc network. For reliable transmission over the wireless network, shortest path is not a good choice to reach its destination[17], it causes delay due to more traffic in at least in one node in that shortest path through out in network.

To manage the traffic, routing protocols are divided into three categories: first is flat routing, in which any path from source to its destination is chosen with hop by hop method, second one is hierarchical routing where routers are grouped together to form hierarchy and third is geographic position assisted routing protocols[8]. Flat routing is divided into reactive and proactive approach. Whole network in AODV is flooded by the RREQ (Route Request) and RREP (Route Reply) packets. This unnecessary flooding through each nodes leads to high traffic on network. To determine the geographical location between source and destination nodes, GPS (Global Positioning System) is used. To reduce the traffic on network it is necessary to reduce the search space for a desired route, for this Location Aided Routing (LAR) [10] protocol is used, this is similar to DSR [12]. In LAR, location information which is obtained from GPS is piggybacked on all messages, so total overhead is decreases.

To minimize the traffic on wireless network another geographical routing protocol is Greedy Perimeter stateless routing (GPSR), which comprises of two types of forwarding: Greedy forwarding and perimeter forwarding. As the mobility of nodes increases, it also increases the breakages in the routes which results in rediscovering the routes in networks. To avoid such traffic and collision, a new process called Velocity AwareProbabilistic discovery model is identified [4]. While constructing routes from source to its destination, this model identifies the unstable nodes (having high mobility) from the network and excludes such nodes from route. To manage the load in Ad Hoc network, density probabilistic scheme [5] distance based probabilistic scheme [21], a position aware counter based scheme [5] were identified for minimization of rebroadcasting process.

To find out the optimal path to any given destination in mobile ad hoc wireless networks, routing protocols consider the path with the minimum number of hops. But, this strategy does not properly balance the traffic load over the Ad-hoc network, and may create congested areas. Due to creation of congested areas it greatly degrades the performance of the routing protocols. In this paper [1], we propose a routing scheme that balances the load of the network by selecting a path based on traffic sizes. In this paper it presents a simulation study to demonstrate the effectiveness of the proposed scheme.

Detailed result of packet-level simulation [2] comparing four multi-hop wireless ad hoc network routing protocols: DSDV, TORA, DSR, and AODV that covers a range of design choices. This paper have extended the OPNET network simulator to accurately model the physical-layer and MAC behavior of the IEEE 802.11 wireless LAN standard, a realistic wireless transmission channel model, and it also gives the simulations results of networks of 50 mobile nodes.

Ad-hoc On Demand Distance Vector Routing (AODV) Paper [14], it is one of the novel algorithm for the operation of such mobile ad-hoc networks. While repairing broken links also, AODV provides loop-free routes over the network. Because the AODV protocol does not require any global periodic routing advertisements, the demand is available on the overall bandwidth to the mobile nodes is substantially less. By this algorithm large number of mobile nodes wishing to form ad-hoc networks can be achieved.

On-demand routing protocol establishes a route from source to a destination node only when needed. However, most of on-demand routing protocols reestablish a new route after a route break. To avoid route breaks, paper [24], propose a new route maintenance algorithm. In this algorithm each intermediate node on an active 
route identifies a danger, if a link breaks, and gives the information to an upstream node and then reestablishes a new route.

Another Paper [18] presents a new routing algorithm called adaptive distance vector (ADV) for mobile, ad hoc networks (MANETs). It is a distance vector routing algorithm that inherits some on-demand properties by varying the size and the frequency of the routing updates in response to the mobility conditions and network load. Using simulations [18] show that ADV outperforms DSR and AODV especially in high mobility of nodes in network by giving significantly lower packet delays and higher $(50 \%$ or more) peak throughputs.

This enhanced version of AODV uses the two methods for traffic analysis on network Packet scheduling algorithm and Weight hop-based scheduling algorithm.

\subsection{Packet scheduling algorithm}

To study the effect of time-to-time delivery of control packets in an on demand routing protocol, we compare a scheduling algorithm that does not distinguish control packets and data packets. It maintains queues in first in first out order which separates all control packets and data packets in network [18].

\subsection{Weight hop-based scheduling algorithm}

It gives higher weight to data packets which have fewer remaining hopes or shorter geographical distances to reach to its destination. For this it uses the concept of weighted round-robin scheduler instead of static priority scheduler to overcome with the problem of starvation. Thus the traffic is gradually reduced from each intermediate nodes and delay for the execution is considerably reduced than the other scheduling algorithm. The reduction in the average delay decreases as the mobility of nodes [2].

\subsection{Tactical Ad-hoc on Demand Distance Vector Routing}

It uses the Query localization process that reduces network traffic .The load checking process is performed by each intermediate node that process a route request. The load of a node is determined by the length of the protocol queue at a node. The method used to select the best route is similar to the procedure in Dynamic Load Aware Routing (DALR) Protocol [15].Before broadcasting the route request packet, intermediate nodes determine the length of its protocol queue, if it is above the threshold value " $\mathrm{t}$ ", it increments the load variable in the packet. Three conditions are specified in [11] to determine the whether the route is better than another route. This method checks the load on each intermediate node and then selects a path to reach its destination from the source node.

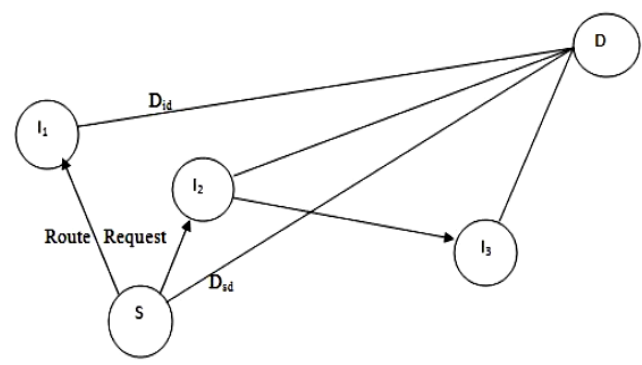

Figure.3. Rebroadcast decision in TAODV

The distance calculated by the source node Dsd (Fig. 3) is included in the route request packet broadcast to its neighbors. The timestamp of the location information (tsd) used to calculate Dsd is also one of the fields in the packet. When a node between the source node and destination node receives the packet, the first action makes a query to its location cache for an entry for the destination node. If an entry is found in location cache, then the timestamp of the entry (tid) is compared with the location information timestamp in the route request packet (tsd). If tid is newer than this tsd, it implies that the intermediate node's location information for the destination is more recent [11].

\section{Proposed Algorithm}

In order to calculate the high throughput path among the existing paths from source to destination, we considered load and delays of total path. If the distance increases delay also increases. In our proposal, we define cut-off conditions to decide the high throughput path and categorize the paths based on throughput.

\subsection{Delay on Transmission}

Nodal processing involves four types of delays namely processing delay, propagation delay, queuing delay, and delay for transmission: 


\subsubsection{Processing Delay (dp)}

When a packet arrives (with all its bits), processing delay is the time consumed to determine the packet header and examine where it should be directed. After processing, the router directs the packets to the queue. This delay is usually in microseconds ( $\mu$ s).

\subsubsection{Queuing Delay (dq)}

Queuing delay is the waiting time in the queue for a packet before it transmits onto a link. The queuing delay of a specific packet will depend on the number of earlier-arriving packets that are queued and waiting for transmission across the link. It is usually of the order milliseconds (ms). In the present work, we consider average queuing delay in our computations.

\subsubsection{Transmission Delay (dt)}

The amount of time required to push (i.e., transmit) all of the packet's bits onto the link is called as transmission delay. This includes the addition of current router information apart from sending acknowledgement signal from current router to the parent router/ downstream router on successful transmission.

\subsubsection{Propagation Delay (dg)}

The time required to propagate from the end of the downstream router to the upstream router is called as propagation delay. It is usually of the order milliseconds. So, the total nodal delay is given by:

$$
\mathrm{Dtn}=\mathrm{dp}+\mathrm{dq}+\mathrm{dt}+\mathrm{dg}
$$

Each of the above delay has a considerable impact on packet transmission. A large processing delay keeps the buffer empty for most of the time; and a large transmission delay fills the queue and subsequently the packets (upcoming) may be lost due to unavailability of bandwidth. Queuing delay is maximum for a proper path selected and minimized for the best path selected. In this work, our focus is more on queuing delay as other delays are mostly hardware dependent.

\subsection{Load}

To find load of path there is need to find number of packet is in queue for every node who are participating in route selection algorithm. When RREQ packet broadcast for route discovery process this packet keep record of every node load and add to its path load. When this RREQ packet reaches to destination, the destination read this value and select path having low load for route establishment as sending RREP packet to lightly loaded path.

This is average value of packets under processing all intermediate nodes in path from source to destination.

\subsection{Route Weight Calculation}

Proposed algorithm compute total delay of path and total load on path by averaging value of all nodes appears between source and destination. Path selection is based on weight of path weight of path is calculated by considering both parameters load and delay equally hence here constant $\mathrm{a}$ and $\mathrm{b}$ introduced any value between 0 and 1 , these are selection parameters such that $a+b=1$. If load is most effective value for path selection such type of networks where network traffic heavily loaded so better to consider load primary selection criteria hence a is greater than $b$ to take more weighting to load than delay. MANET is spread up long distance for larger coverage area so delay could be play crucial role for path selection because of more delay. If network is normally setup for delay and load so both decision parameters are equal to 0.5 .

$$
\mathrm{Wp}=\mathrm{a} * \mathrm{Lp}+\mathrm{b} * \mathrm{Dp}
$$

Where $\mathrm{Wp}$ is weight of current path, Lp is load of path according all nodes cover this path and delay is average delay of all nodes cover within current path. $\mathrm{a}$ and $\mathrm{b}$ are constant between 0 and 1 such that $\mathrm{a}+\mathrm{b}=1$.

\subsection{Example}

For example from this Figure: node 4 act as a source node and node 2 act as a destination node. Here to identify the best route selection in terms of minimum delay and load is calculated as follows. 


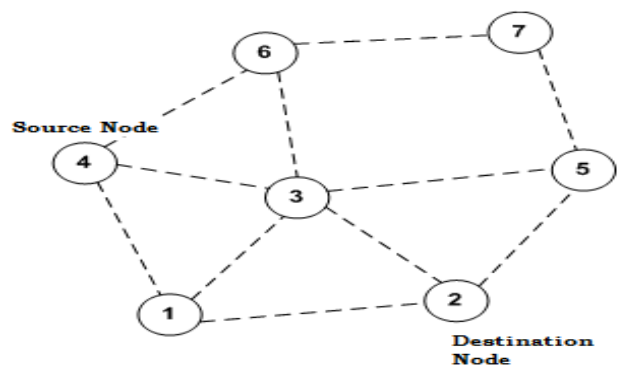

Figure: Simple Mobile Ad-hoc Network

In mobile Ad-hoc network consider the load and delay associated with each route is given as:

Node 1: load $=45$ and delay $=30$

Node $2: \operatorname{load}=40$ and delay $=25$

Node 3: 1 load $=50$ and delay $=60$

Node 4: 1 load $=40$ and delay $=20$

Node 5: 1 load $=50$ and delay $=25$

Node 6: 1 load $=45$ and delay $=35$

Node 7: load $=60$ and delay $=40$

From source node to destination node there many numbers of routes available. We consider only four routes to reach destination node such as: 4-1-2, 4-3-2, 4-1-3-2 and 4-3-1-2.

Now the proposed algorithm calculates the best route from source to destination in terms of weight calculation. For this the average load and delay is calculated in each route. Thus the average weight associated with each route is given as:

Route I: 4-1-2:

$\mathrm{W} 1=(0.5 * 40+0.5 * 20)+(0.5 * 45+0.5 * 30)+(0.5 * 40+0.5 * 25)=100$

Route II: $4-3-2$ :

$\mathrm{W} 1=(0.5 * 40+0.5 * 20)+(0.5 * 50+0.5 * 60)+(0.5 * 40+0.5 * 25)=117.5$

Route III: 4-1-3-2:

$\mathrm{W} 1=(0.5 * 40+0.5 * 20)+(0.5 * 45+0.5 * 30)+(0.5 * 50+0.5 * 60)+(0.5 * 40+0.5 * 25)=163$

Route IV: 4-3-1-2:

$\mathrm{W} 1=(0.5 * 40+0.5 * 20)+(0.5 * 50+0.5 * 60)+(0.5 * 45+0.5 * 30)+(0.5 * 40+0.5 * 25)=155$

Thus the minimum weight is obtained from route is $1-4-2$, which is optimal path from source node 4 to destination node 2 . So route number 1 will be most efficient for transmitting data as having lowest weight. This selection process considering load and delay both equally. By putting $\mathrm{a}=1$ and $\mathrm{b}=0$ this method consider only load for path selection and not delay hence this scheme becomes TAODV. From this algorithm the traffic present on each node in term of its load and delay in transmission is also obtained. So the congestion on the network can be removed by recognizing the path which is highly loaded.

\section{Algorithm}

Overall algorithm given in above section can be summaries as following steps for efficient route discovery.

\subsection{NS-3 Simulations}

1. Design Wi-Fi topology in NS-3 simulator.

2. Install MAC, IP and Application protocol on each node.

3. Select MANET routing protocol AODV start simulation

4. For each node i

a. do

b. RREQ start (in case of AODV)

c. Flooding of RREQ

d. Build routing table (AODV. route file)

i) Collect behaviors statistics such as No. of packet transmitted, received, delay time, modified etc.

ii) Serialize into. $\mathrm{xml}$ file format

\subsection{Route Selection}

1. Input .xml data file Generated by NS-3 to JAVA Simulator

2. Extract information

a. For each node i repeat

b. Input .xml onto DOM (Data Object Module) 
c. Calculate average load and delay for every node i.

d. Draw as their coordinate value all nodes in Network display.

3. Basis of route total load and total delay calculate Weight for every path as $0.5 *$ load $+0.5 *$ delay Select best path according minimum weight.

4. Displayed selected result path with all intermediate nodes in path.

\section{Simulation AND ReSUlts}

The performance and capabilities of the proposed broadcast schemes are examined and investigated using NS-3.12 as the simulation platform. For each data points in all the figures, at least 30 experiments are used, each one represents different network topology with $95 \%$ confidence intervals. The number of nodes in the network was chosen between 20 to 200 nodes for all scenarios. The nodes are placed in $400 \mathrm{~m} \mathrm{X} \mathrm{400m} \mathrm{square}$ area. The random waypoint model is used as the mobility model. In this model, mobile nodes move freely and randomly without boundary restrictions. The application layer at each node generates CBR traffic. Maximum nodes speed varies between $10 \mathrm{~m} / \mathrm{s}$ to $40 \mathrm{~m} / \mathrm{s}$. Due to its high capability in MANETs. AODV routing has been adopted in our experiments. Following simulation parameters we have used under NS-3 test bed.

TABLE I. SimUlation PARAMETER

\begin{tabular}{|l|l|}
\hline \multicolumn{1}{|c|}{ Parameter } & \multicolumn{1}{c|}{ Value } \\
\hline Mobility Model & Random Waypoint model \\
\hline Nodes (Wi-Fi Node's) & 20 to 50 nodes \\
\hline Simulation Time & $100 \mathrm{~s}$ \\
\hline Packet Size & 1000 bytes \\
\hline Sink Nodes & 17 \\
\hline Update Interval & $15 \mathrm{~s}$ \\
\hline Node Speed & Up to $10 \mathrm{~m} / \mathrm{s}$ \\
\hline Traffic Model & Constant Bit Rate \\
\hline Settling Time & 6 \\
\hline Routing Protocols & AODV \\
\hline
\end{tabular}

\subsection{Experimental result}

We have created one mobile Ad-hoc network which consists of 25 nodes having the transmission range as 100. By creating this through NS-3 simulator we generate .xml file which is taken as input to the JAVA simulator. Following figure shows the calculation of route from source to destination. Out of 25 nodes any node may act as the source node and destination node.

In this result we have the best route in term of its minimum load and require minimum delay, which is shown by the weight associated with it. Here in the result shown in Fig.4, node 5 act as the source node and node 21 as the destination node, both nodes are represented by the red circle.

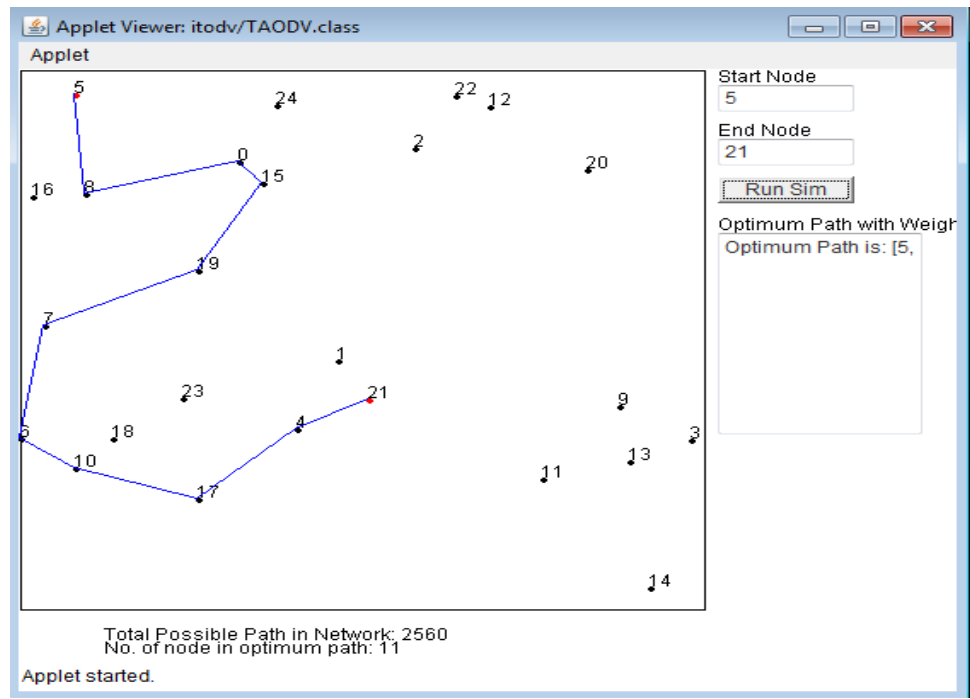

Figure.4. Result showing optimal path from source node to destination 


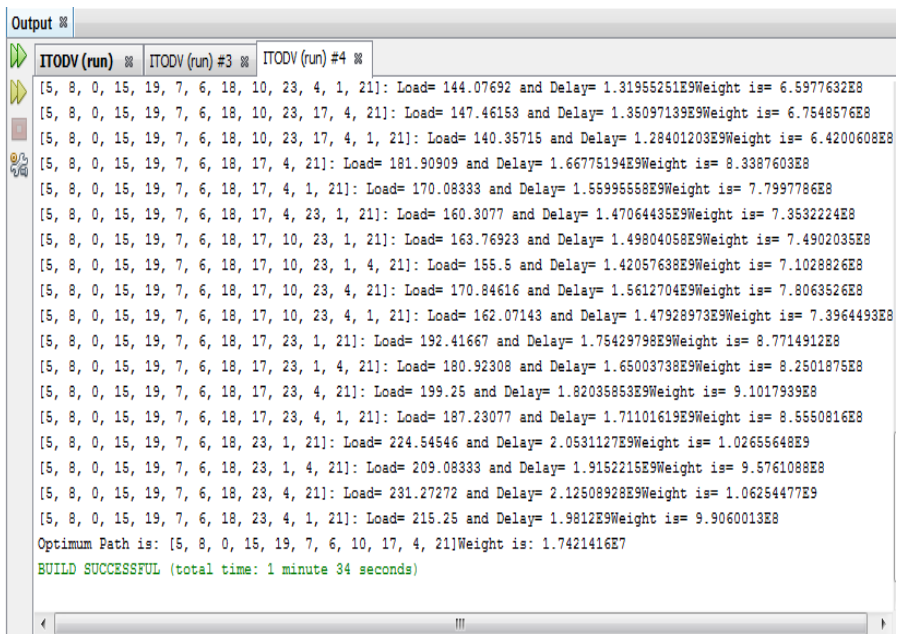

Figure.5. Result showing all possible routes from source to destination with weight

Fig. 4 and Fig. 5 shows there are 2560 routes available from source to the destination node and the best route among these route is $[5,8,0,16,19,7,6,10,17,4,21]$. The best route is calculated by the equation (1), having weight associated with this route is $17421416 \mathrm{E} 7$ which is the minimum weight among all routes.

\subsection{Average end-to-end delay}

This is the overall average delay required by a packet to travel from source node to its destination node. The average total path end-to-end results given by simulation observe that Efficient TAODV packet delay is less delay than TAODV in all of the scenarios considered for the simulations in Figure 6. The delay is significantly lower in the 16 node network configuration where there is a considerable network traffic load. In both, the high mobility and the low mobility scenario sets, the delay shown by Efficient TAODV is at least $200 \mathrm{~ms}$ lower.

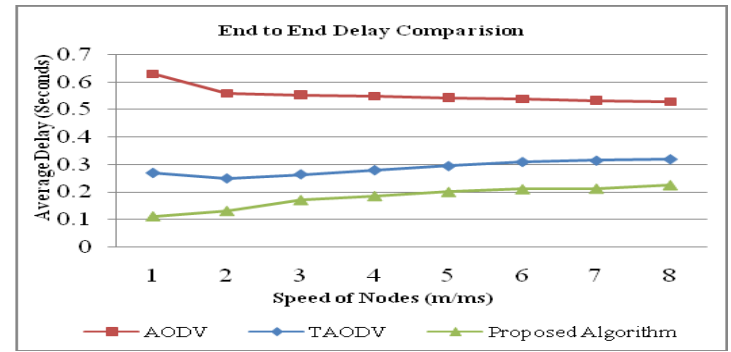

Figure: 6. Delay performance with TAODV scheme.

\subsection{Comparative study of Delay}

It shows behavior for different constant factor value according to proposed weight calculation to select optimum path is following:

$$
\mathrm{W}=\mathrm{A} * \text { total path Load }+\mathrm{B} * \text { total path delay }
$$

Where $\mathrm{A}$ and $\mathrm{B}$ are constant and these are decided according to network condition of MANET.

When value changes of parameters A and B, the fluctuations in delay appears. We have calculated the weight for the different scenario. In first case we have consider only load during packet transmission, in second scenario only delay is considered, and in third one both delay and load is considered with equal value. The different weight value associated with each scenario is shown in Table.2.

Table.2. Comparison Delay for various factors for constant A and B

\begin{tabular}{|c|c|c|c|}
\hline \multirow{2}{*}{ Node Speed $(\mathrm{m} / \mathrm{s})$} & $\begin{array}{l}\text { Delay Factor=0 } \\
\text { \&Load Factor=1 }\end{array}$ & $\begin{array}{l}\text { Delay Factor=1.0 } \\
\text { \&Load Factor=0 }\end{array}$ & $\begin{array}{c}\text { Delay Factor }=0.5 \\
\text { \&Load Factor=0.5 }\end{array}$ \\
\cline { 2 - 4 } & $\mathrm{A}=0, \mathrm{~B}=1.0$ & $\mathrm{~A}=1.0, \mathrm{~B}=0$ & $\mathrm{~A}=0.5, \mathrm{~B}=0.5$ \\
\hline 1 & 0.345 & 0.27 & 0.11 \\
\hline 2 & 0.34 & 0.25 & 0.13 \\
\hline 3 & 0.32 & 0.265 & 0.17 \\
\hline 4 & 0.29 & 0.28 & 0.185 \\
\hline 5 & 0.28 & 0.295 & 0.2 \\
\hline 6 & 0.275 & 0.31 & 0.21 \\
\hline 7 & 0.275 & 0.315 & 0.212 \\
\hline 8 & 0.275 & 0.32 & 0.225 \\
\hline
\end{tabular}


From Fig.7. We can observe that if nodes are moving with very slow speed then every node has more packets to deliver due to its static position and better route discovery. Hence is good to give more importance to load which is key factor for efficient path calculation. Whereas if nodes are moving at fast speed so any node doesn't have lot of packet as moving quickly from its current position so here delay of node is good criteria for path selection. If consider both parameter equally so it will give better performance in every situation.

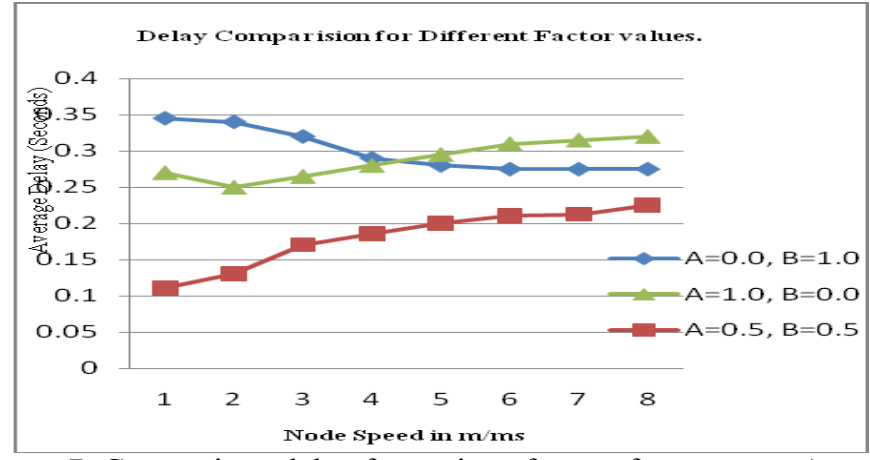

Figure.7. Comparison delay for various factors for constant A and B

\section{CONCLUSION}

The IEEE 802.11 standards support multi-rate capability in MANETs. There are several routing protocols in the literature to select a routing path that optimizes the throughput. In this work, we study the quality of service maintained during routing through multi rate MANETs and enhanced the TAODV approach by augmenting the load and delay parameter in the model. The presented model is a simple but effective, which reveals that the quality of service is not only depends on only load but also on delay. Also, our model maintains the multiple routes with ranking and routing decision based on the weight, which can help during failure of any route.

During low mobility, the average delay is dominated by network congestion due to data traffic. During high mobility, it is dominated by route changes in the simulation results. Our scheduling algorithms that give higher weight to data packets with smaller numbers of hops or shorter geographic distances to their destinations reduce average delay significantly without any additional control packet exchange. The weighted-hop scheduling algorithm is used for modified AODV. Result show considerably smaller delay than the other scheduling algorithms.

\section{References}

[1] Mahesh KM, and Das SR. On-demand multipath distance vector routing in Ad hoc networks. Ninth International Conference on Network Protocols, Washington D.C., USA, 2001, 14-23.

[2] Dhirendra Kumar Sharma, Chiranjeev Kumar and Sandeep Jain, Neeraj Tyagi, “An Enhancement of AODV Routing Protocol for Wireless Ad Hoc Networks”, 1st Int'l Conf. On Recent Advances in Information Technology| RAIT-2012 | IEEE, 2012

[3] Syed Jalal Ahmad, V.S. K. Reddy, A. Damodaram and P. Radha Krishna, "Efficient Path Estimation Routing Protocol for QoS in Long Distance MANETs", IEEE, 2012.

[4] Mustafa Bani Khalaf, Ahmed Y. Al-Dubai and Mourad Abed, "New Velocity Aware Probabilistic Route Discovery Schemes for Mobile Ad hoc Networks", IEEE, 2012.

[5] P. Y. Taifei Zhao, Xizheng Ke, "Position and velocity aided routing protocol in mobile ad-hoc networks, " International Journal of Digital Content Technology and its application, vol. 4, pp. 101-109, 2010.

[6] Anh Tai Tran, Myung Kyun Kim , "A real-time communication protocol considering load balancing in Adhoc Network", IEEE, 2013.

[7] Mohd. Ayash, Mohd. Mikki, Kangbin Yim, “Improved Aodv Routing Protocol to Cope with High Overhead in High Mobililty MANETs “, Sixth Int. Conference on Innovative Mobile and Internet Services in Ubiquitous Computing, 2012.

[8] Y. Hassan, M. Abd El-Aziz, and A. Abd El-Radi, "Performance evaluation of mobility speed over MANET routing protocols," International Journal of Network Security, November 2010,Vol. 11, No.3, pp.128-138.

[9] A. Mohammad, M.ould-khaoua, L.Mackenzie, abdulai "Dynamic probabilistic counter based routing in mobile adhoc network, "2nd international conference on Adaptive Science Technology, Jan 2009.

[10] P. Y. Taifei Zhao, Xizheng Ke, “Position and velocity aided routing protocol in mobile ad-hoc networks, " International Journal of Digital Content Technology and its application, vol. 4, pp. 101-109, 2010.

[11] M. uddin, Abdul Rahman, A. Alarifi, Mohd. Talha, A. Shah, Mohsin Iftikhar and A. Zomaya, "Improving performance of Mobile adhoc networks using efficient Tactical on demand distance vector (TAODV) routing algorithm, vol.8, international journal of innovation and control, June 2012.

[12] Ali Khosrozadeh,Abolfazl Akbari,Maryam Bagheri and Neda Beikmahdavi "NEW AODV ROUTING PROTOCOL WITH BREAK AVOIDANCE",IEEE,International Symposium on Computer Science and Society,2011.

[13] S. Nanda, and R. Gray, "Multipath location aided routing in 2D and 3D," Wireless Communications and Networking Conference, 2006, 3-6 Apr., IEEE Vol. 1, pp.311-317.

[14] Abdulrahman H. Altalhi Golden G. Richard, III "Load-Balanced Routing through Virtual Paths:Highly Adaptive and Efficient Routing Scheme for Ad Hoc Wireless Networks",IEEE,2004

[15] Y. KO and N. H. Vaidya, “Location-Aided Routing (LAR) in mobile Ad-hoc networks,” Wireless Networks, 2000, pp.307-321. 
[16] J. Broch, D.A. Maltz, D.B. Johnson, Y. Hu and 1.Jetcheva, "A Performance Comparison of Multi-hop Wireless Ad Hoc Network Routing Protocols," in Proceedings of ACM MOBICOM'98, Dallas, Texas, USA, October 1998.

[17] Peter P.Pham and Sylvie Perreau, "Performance analysis of reactive shortest path and multipath routing mechanism with load balance”, in Proc. Of the IEEE INFOCOM, march 3 April, 2003.

[18] C.E. Perkins and E.M. Royer, “Ad-Hoc on-Demand Distance Vector Routing”, Proceedings of IEEE WMCSA'99, New Orleans, LA. (Feb.1999) pp: 90-100.

[19] IndraWidjaja, Anwar Elwalid. MATE: MPLS Adaptive Traffic Engineering, draft-widjaja-mpls-mate-01. txt, Internet Draft [ DB/ $(D B / O L)][S]$. october , 1999 .

[20] Amjad Ali, Wang Hiugiang “Node Centric Load Balanncing Routing Protocol for Mobile Ad Hoc Networks “, Proceeding of International MultiConference of Engineers abd computer Scientist 2012, Vol I, March 2012, Hong Kong.

[21] P. Pranitha, G. Swamy, and M. Aakunuri,"'A review on enhanced GPSR protocol for wireless sensor networks," Computer Engineering and Intelligent Systems, 2011, Vol. 2, No. 4.

[22] O. Tonguz, N. Wisitpongphan, J. Parikh, F. Bai, P. Mudalige, and V. Sadekar, "On the broadcast storm problem in ad hoc wireless networks," in 3rd International Conference on Broadband Communications Networks and Systems, pp. 1 -11, 2006.

[23] Douglas S.J., Daniel Aguyao, John Bickrt and Robbert Morris, "A high Throughput path metric for multihop wireless routing” in Proc. Of 9th annual international conference on Mobile computing and networking, ACM, pp 134-146, 2003

[24] Amita Rani, Mayank, Dave, "Load Balancing Routing Mechanism for Mobile Ad Hoc Networks “, Int. J. Communications Network and System Sciences, 2009, 7, 627-635.

[25] A.Rajaram and S.Palaniswami. A trust based cross layer security protocol for ad hoc networks. International Journal of Computer Science And Information Security, 6(1), 2009. [cited at p. 8] 